Une expérience professionnelle à l'étranger est-elle payante en début de carrière pour les diplômés de l'enseignement supérieur?

\title{
Cathy Perret
}

\section{(2) OpenEdition}

Journals

Édition électronique

URL : https://journals.openedition.org/remi/4849

DOI : 10.4000/remi.4849

ISSN : $1777-5418$

Éditeur

Université de Poitiers

\section{Édition imprimée}

Date de publication : 1 décembre 2008

Pagination : 209-224

ISBN : 978-2-911627-50-7

ISSN : 0765-0752

Référence électronique

Cathy Perret, « Une expérience professionnelle à l'étranger est-elle payante en début de carrière pour les diplômés de l'enseignement supérieur? ", Revue européenne des migrations internationales [En ligne], vol. 24 - n³ | 2008, mis en ligne le 01 décembre 2011, consulté le 14 avril 2022. URL : http:// journals.openedition.org/remi/4849; DOI : https://doi.org/10.4000/remi.4849 


\title{
Une expérience professionnelle à l'étranger est-elle payante en début de carrière pour les diplômés de l'enseignement supérieur?
}

\author{
Cathy PERRET*
}

Bien qu'aucune estimation officielle n'existe, l'idée répandue est que de plus en plus de jeunes prennent la décision d'émigrer pour trouver un emploi (FrançoisPoncet, 2000). Reposant à l'origine sur le constat d'une expatriation croissante des jeunes chercheurs, laquelle est associée à une " fuite des cerveaux », néfaste au développement économique du pays en cas de non retour, le débat sur les causes et conséquences des départs s'est élargi à l'ensemble des diplômés français. Mais, les jeunes y trouvent-ils un intérêt en début de carrière professionnelle ? Présentées comme une expérience enrichissante pour les jeunes, de telles migrations s'inscrivent également dans un contexte plus général de la construction attendue d'un marché du travail à l'échelle européenne, considéré comme nécessaire à la compétitivité européenne (Haug et Tauch, 2001).

En ce qui concerne les étudiants, et notamment les étudiants français, il existe un certain nombre de travaux sur leurs mobilités à l'étranger dans le cadre des programmes Erasmus (Commission européenne 2000 ; Cattan, 2004, 2007). Certains travaux abordent ainsi les questions de l'intérêt de telles mobilités telles qu'elles sont perçues par les étudiants avant ou après cette expérience (Murphy-Lejeune, 2005 ; Papatsiba, 2003 ; Pichon, 1999). D'autres travaux s'intéressent à l'effet de ces

* Ingénieur de recherche, CIPE (Centre d'Innovation Pédagogique et d'Evaluation), Chercheur associé à UMR CNRS 5225 IREDU, Université de Bourgogne, Pôle AAFE - Esplanade, Erasme, BP 26513, F - 21065 Dijon cedex, cathy.perret@u-bourgogne.fr 
mobilités à l'étranger sur le devenir professionnel (Teichler et Kerstin, 2007). Toutefois, rares sont les travaux qui s'intéressent aux mobilités à l'étranger entreprises par les jeunes une fois leurs études terminées. En fait, pour les diplômés français, seule la mobilité internationale des titulaires d'un doctorat a fait l'objet d'explorations (Recotillet, 2007). Parallèlement, jusqu'à présent, les analyses de l'insertion professionnelle des diplômés de l'enseignement supérieur s'intéressent seulement au devenir professionnel des jeunes sur le marché du travail national. Elles abordent rarement les mobilités géographiques, et encore moins les migrations internationales. En effet, peu d'enquêtes comportent des informations géographiques fines, alors que les années qui suivent les années de formation apparaissent comme une période particulièrement propice aux déplacements. Or l'enquête «Génération 98 » du Céreq nous offre l'opportunité de tenter de répondre à certaines questions concernant les jeunes ayant eu un emploi à l'étranger à l'issue de leurs études et rentrés en France. Quel est donc le profil de ces jeunes ? Pourquoi partent-ils?

Cet article vise ainsi à mieux comprendre les départs temporaires à l'étranger des diplômés de l'enseignement supérieur en début de carrière lorsqu'ils sont associés à une prise d'emploi. Il se décompose en quatre parties. La première et la deuxième présentent respectivement des informations sur ces migrations temporaires et sur les emplois occupés par les jeunes français à l'étranger. La troisième explore les déterminants de cette migration à l'étranger en examinant les caractéristiques scolaires et sociales des partants et non partants. Enfin, la quatrième partie apporte des éléments de réponse à la question « une expatriation professionnelle temporaire est-elle toujours synonyme de conditions d'emploi plus favorables qu'en France? ».

\section{LES MOBILITÉS INTERNATIONALES TEMPORAIRES DES DIPLÔMÉS DE L'ENSEIGNEMENT SUPÉRIEUR FRANÇAIS EN PHASE D'INSERTION PROFESSIONNELLE}

Peu nombreuses, les sources d'informations sur les Français à l'étranger permettent difficilement de repérer les diplômés de l'enseignement supérieur partis acquérir une expérience professionnelle à l'étranger et d'apprécier les déterminants et effets de telles mobilités géographiques. L'enquête «Génération 98 » du Céreq dédiée au devenir des jeunes à la fin de leurs études, permet de repérer une partie des migrations internationales des diplômés de l'enseignement supérieur français : celles des jeunes insérés sur le marché du travail national trois ans après la fin de leurs études, sachant que l'expatriation professionnelle est estimée par le fait d'avoir occupé un emploi à l'étranger après la fin des études. Précisons qu'il s'agit d'une migration temporaire (moins de trois ans) et que tous les jeunes émigrants ne sont pas recensés (cf. encadré 1). À cet égard, il est important de préciser que seuls les jeunes résidant en France à la date de l'enquête (c'est-à-dire trois ans après leur sortie de formation) sont intégrés dans cette enquête. Il existe ainsi un biais important lié aux jeunes expatriés qui ne sont pas rentrés en France au bout de trois ans. 
Encadré 1 : Mobilités internationales des diplômés : les possibles et les limites

\section{L'enquête}

Les données utilisées sont issues de l'enquête "Génération 98 » réalisée par le Céreq (Centre d'études et de recherche sur les qualifications) au printemps 2001 (Céreq, 2002). L'enquête a touché plus de 30000 sortants de l'enseignement supérieur parmi les 367000 jeunes qui avaient quitté pour la première fois le système supérieur d'éducation. Elle couvre l'ensemble des sortants des universités, du premier au troisième cycle, des IUFM ainsi que les jeunes issus des écoles d'ingénieurs et de commerce, d'art et d'architecture, de la santé et du social, qu'ils aient ou non obtenu le diplôme correspondant à la classe de sortie. Sont exclus du champ de l'enquête, les formations dépendant du ministère de la Jeunesse et des Sports, les écoles d'ingénieurs sous tutelle du ministère de la Défense, les écoles de notariat, les écoles juridiques, les écoles normales supérieures, les élèves sortis non diplômés des écoles de l'enseignement supérieur, les étudiants de nationalité étrangère issus de l'Université. Elle ne concerne que des populations de jeunes de nationalité française et âgés de moins de 35 ans lors de leur sortie de formation.

\section{Les diplômés de l'enseignement supérieur sur le marché du travail national en} 2001

Seules les situations des jeunes résidant en France à la date de l'enquête peuvent être appréciées, compte tenu de la construction de l'enquête (les personnes résidant en France sont interrogées). Les rares personnes résidant à l'étranger ou dans les DOM-TOM en 2001 apparaissant sont en fait principalement des personnes qui se trouvaient exceptionnellement sur le territoire national (vacances chez les parents principalement) et sont nullement représentatives des situations de tels jeunes partis à l'étranger. Il est important de souligner que cette enquête n'interroge pas les jeunes demeurant à l'étranger à la date de l'enquête. Ces derniers n'étant pas inclus dans le champ de l'enquête "Génération 98 ", il n'est donc pas possible de produire une estimation fiable des taux de départs à l'étranger dans les trois ans qui suivent la fin des études, ni de comparer les caractéristiques de ces expatriés avec les partants revenus en France en 2001 ou avec les sédentaires.

Dans la mesure où nous nous intéressons à l'insertion professionnelle des diplômés de l'enseignement supérieur français, seuls ont été retenus les jeunes ayant obtenu un diplôme supérieur au baccalauréat et ayant eu au moins un emploi dans les trois ans qui suivent la fin de leurs études. Ce sont des jeunes insérés sur le marché du travail national trois ans après la fin de leurs études (au chômage ou en emploi en mars 2001 et résidant en France à cette date). Par ailleurs, sont exclus de notre échantillon les jeunes dont le lieu d'emploi en 2001 ou le lieu du premier emploi ne sont connus, ainsi que les jeunes résidant en France en 2001 et travaillant à l'étranger en 2001 parce que le travail 
frontalier n'est pas l'objet de cette recherche. Notre étude est ainsi réalisée à partir d'un échantillon de près de 21700 diplômés, représentant environ 250000 jeunes qui avaient quitté pour la première fois le système supérieur d'éducation en 1998.

\section{Une expatriation professionnelle temporaire}

Compte tenu du champ de l'enquête, seules sont appréciées les mobilités intervenues au cours des trois ans qui suivent la fin des études et caractérisées par un retour en France. Il s'agit donc de migrations temporaires. Dans cette enquête, le calendrier des mobilités est imparfait puisque les seules informations « géo-référencée » sont les lieux de résidence en 1998 et 2001, la région de formation en 1998 et la localisation des emplois obtenus au cours des trois premières années de vie active. Les migrations à l'étranger peuvent ainsi seulement être connues à partir de la description des emplois. À ce stade de notre étude, les mobilités internationales temporaires sont estimées à partir du pays d'obtention du premier emploi à la sortie de formation (emplois de vacances exclus), sachant que le premier emploi est un élément structurant de la trajectoire professionnelle des jeunes. L'examen des mobilités temporaires fait abstraction des mobilités multiples pouvant avoir eu lieu entre la sortie de formation et le printemps 2001. Ces choix permettent d'analyser plus simplement les déterminants des mobilités en éliminant le problème de l'enchaînement des différents emplois et notamment les questions de promotion professionnelle susceptible d'intervenir dans le cadre d'une mobilité internationale. Les mobilités temporaires de ces jeunes sont faiblement sousestimées dans la mesure où les jeunes partant après avoir eu un premier emploi en France sont peu nombreux (quelques dizaines). Reste qu'il est impossible d'associer strictement la date de commencement de ce premier emploi à la date de la mobilité, puisqu'on ne sait pas si les jeunes sont partis à l'étranger avant d'occuper cet emploi ou après.

Dans notre étude, les diplômés de l'enseignement supérieur français présents sur le marché de l'emploi national trois ans après la fin de leurs études ont rarement eu une expérience professionnelle à l'étranger. En effet, seuls $2 \%$ des jeunes de notre échantillon ont eu un premier emploi à l'étranger.

Ces jeunes privilégient des destinations proches dans des pays limitrophes au territoire français. Comme l'ensemble de la population française immatriculée à l'étranger (Gentil, 2003), la majorité des mobiles ont une première expérience professionnelle dans l'Union européenne ou en Suisse $(57 \%)$ et participent ainsi effectivement à la construction du marché du travail européen (cf. déclaration de Bologne). Seul un jeune sur dix a traversé l'Atlantique. Toutefois, les conclusions tirées de ces estimations doivent être fortement relativisées compte tenu des biais d'échantillonnage existants. En effet, faut-il voir dans cette faible proportion le fait que peu de jeunes choisissent de partir en Amérique du Nord, ce qui va l'encontre de l'idée d'une 
« fuite des cerveaux » aux États-Unis ? ou encore la conséquence que les mobilités des diplômés de l'enseignement supérieur ne sont pas seulement le fait des personnels scientifiques et technologique pour lesquels les États-Unis font figure de pôle mondial d'attraction ? ou bien plutôt n'est pas la conséquence que les jeunes partis outreatlantique sont exclus de l'analyse car ils ne sont pas revenus en France trois ans après la fin de leurs études? (cf. tableau 1).

Tableau 1 : Les zones d'accueil des jeunes ayant effectué une expatriation professionnelle temporaire

\begin{tabular}{|l|c|c|}
\hline Zones & Effectifs & \% \\
\hline Europe occidentale & 2139 & $57 \%$ \\
\hline Amérique du Nord & 436 & $12 \%$ \\
\hline Autres & 1207 & $32 \%$ \\
\hline Total & 3782 & $100 \%$ \\
\hline
\end{tabular}

Source : « Génération 98 », Céreq 2001.

Près de six diplômés sur dix de l'enseignement supérieur français choisissant de franchir nos frontières lors de leur première expérience professionnelle sont des jeunes femmes. Ces dernières sont ainsi légèrement plus nombreuses chez les migrants que chez les jeunes restant au pays (57\% contre $54 \%$ ). À la fin de leurs études, âgés en moyenne de 24 ans, ils étaient plus de quatre sur dix à vivre en couple et parmi ces derniers une minorité avait déjà des enfants $(21 \%)$. Ce sont des jeunes qui sont plus fréquemment issus de familles favorisées : $49 \%$ d'entre eux sont des fils et filles de cadres contre $35 \%$ en moyenne (cf. tableau 2).

Ce sont les jeunes issus des formations de Lettres Sciences humaines et gestion qui sont les plus nombreux parmi les expatriés ayant une expérience professionnelle à l'étranger (28\% des partants). Il faut sans doute y voir la conséquence directe des filières comme LCE (Langues et civilisations Etrangères) ou LEA (Langues Étrangères Appliquées) dont les étudiants sont davantage préparés à travailler dans un environnement international. Ainsi, à l'image des sortants des écoles de commerce, ils sont proportionnellement plus nombreux parmi les migrants que parmi les sédentaires. Un quart des migrants sont diplômés d'un troisième cycle scientifique ou d'une école d'ingénieurs. La main-d'œuvre scientifique et technologique de haut niveau de formation forme ainsi un des contingents les plus importants de migrants auquel peut être associé des transferts de connaissances scientifiques et technologiques entre les pays.

Dans sept cas sur dix, ces jeunes ont effectué au moins un stage professionnel durant leurs études. Plus fréquemment, issus des filières sélectives (écoles de commerce ou d'ingénieur) et de formation de troisièmes cycles, les expatriés ont logiquement eu un meilleur parcours scolaire. Ainsi, seuls $17 \%$ d'entre eux ne figuraient pas parmi les meilleurs élèves du secondaire puisqu'ils n'ont pas obtenu leur baccalauréat à l'heure contre $39 \%$ pour les sédentaires. 
Tableau 2 : Caractéristiques socio-démographiques et scolaires des diplômés de l'enseignement supérieur insérés sur le marché du travail national (en \%)

\begin{tabular}{|c|c|c|}
\hline & $\begin{array}{c}1^{\text {ère }} \text { expérience } \\
\text { professionnelle } \\
\text { à l'étranger }\end{array}$ & $\begin{array}{c}1^{\text {ère }} \text { expérience } \\
\text { professionnelle } \\
\text { en France }\end{array}$ \\
\hline $\begin{array}{l}\text { Caractéristiques socio-démographiques } \\
\text { Femme } \\
\text { Âge médian à la fin des études } \\
\text { Origine sociale } \\
\% \text { père ou mère cadre } \\
\text { Situation familiale à la fin des études } \\
\% \text { en couple } \\
\% \text { ayant des enfants parmi } \\
\text { ceux qui sont en couple }\end{array}$ & $\begin{array}{l}43 \\
21\end{array}$ & $\begin{array}{l}48 \\
27\end{array}$ \\
\hline $\begin{array}{l}\text { Caractéristiques scolaires } \\
\text { Niveau et spécialité de formation } \\
1^{\text {er }} \text { cycle (DEUG, DEUST, BTS-DUT...) } \\
\text { 2d cycle LSH, gestion } \\
\text { 2d cycle maths, sc, tech } \\
3^{\text {ème }} \text { cycle LSH, gestion } \\
3^{\text {ème }} \text { cycle maths, sc, tech } \\
\text { Écoles commerce } \\
\text { Écoles d'ingénieurs } \\
\text { Changement de région entre la } 6^{\text {ème }} \text { et la } \\
\text { fin des études } \\
\text { Baccalauréat obtenu } \\
\text { À l'heure } \\
\text { En retard } \\
\text { En avance } \\
\text { Expérience professionnelle pendant les études }\end{array}$ & $\begin{array}{l}24 \\
28 \\
2 \\
11 \\
14 \\
10 \\
11 \\
50\end{array}$ & $\begin{array}{c}51 \\
23 \\
4 \\
9 \\
5 \\
3 \\
5\end{array}$ \\
\hline
\end{tabular}

Source : « Génération 98 », Céreq 2001.

Par ailleurs, la moitié des expatriés a changé de région durant sa formation. $\mathrm{Ce}$ sont ainsi des jeunes qui ont plus souvent eu une expérience de mobilité que les autres diplômés de l'enseignement supérieur (seulement un peu plus d'un tiers pour les jeunes restés en France dans les trois ans qui suivent la fin de leurs études ont changé de région entre la $6^{\text {ème }}$ et la fin de leurs études).

\section{LES EMPLOIS À L'ÉTRANGER DES JEUNES INSÉRÉS SUR LE MARCHÉ DU TRAVAIL NATIONAL}

Le premier emploi de ces jeunes à l'étranger a duré 9 mois en moyenne et a débuté dans le mois suivant la fin des études. Une infime minorité de jeunes a eu un emploi de plus de deux ans (5\% des jeunes). En outre, l'enchaînement de différents emplois à l'étranger reste exceptionnel pour ces jeunes qui sont de retour sur le marché du travail français en 2001. 
Dans neuf cas sur dix, ils ont travaillé à temps plein et sont rarement passés par des structures d'aides à la recherche d'emploi pour décrocher cet emploi (ANPE, APEC...) ( $2 \%$ contre $10 \%$ en moyenne). Expatriés et jeunes s'insérant directement sur le territoire national sont employés par des entreprises de taille équivalente et dans huit cas sur dix dans le secteur des services.

Dans plus de six cas sur dix, ces jeunes se déclarent satisfaits de cette première expérience professionnelle en estimant qu'elle correspondait à leur niveau de compétences $(64 \%$ contre $56 \%$ pour les jeunes restés en France). Cette satisfaction se combine avec des conditions d'emploi favorables aux jeunes expatriés en termes de statut et de salaires, et ce quel que soit le niveau de formation considéré (cf. tableau 3). En effet, plus de la moitié des jeunes qui se sont tournés vers l'étranger à la fin de leurs études ont obtenu un poste de cadre dès leur premier emploi contre en moyenne seulement le quart des diplômés de l'enseignement supérieur. Compte tenu de la nature des emplois occupés, il apparaît logique que pour leur premier emploi, ces jeunes expatriés bénéficient en moyenne de salaires plus élevés que leurs homologues s'insérant directement sur le territoire national (1 400 euros mensuels nets primes incluses à l'embauche contre 1100 euros). Faut-il y voir dans ces différences, le seul effet de l'existence de marchés du travail nationaux différents ou l'impact d'une meilleure reconnaissance des compétences des diplômés de l'enseignement supérieur à l'étranger ? Cet avantage salarial est valable pour tous les diplômés, à l'exception des diplômés des écoles de commerce et d'ingénieurs qui bénéficient de salaires équivalents à l'étranger et en France.

Tableau 3 : Une première expérience professionnelle plus favorable en termes de statut et de salaires

\begin{tabular}{|l|c|c|c|c|}
\hline & $\%$ cadre & $\%$ Fonctionnaire & $\%$ CDI & $\begin{array}{c}\text { Salaire médian } \\
\text { à l'embauche* }\end{array}$ \\
\hline $1^{\text {er }}$ emploi à l'étranger & 52 & 1 & 32 & 1400 \\
\hline $1^{\text {er }}$ emploi en France & 25 & 6 & 32 & 1100 \\
\hline Ensemble & 25 & 6 & 32 & 1100 \\
\hline
\end{tabular}

* Salaires d'embauche nets primes incluses pour les jeunes en emploi à temps plein.

Source : « Génération 98 », Céreq 2001.

Contrairement à une idée reçue, si ces emplois à l'étranger sont synonymes d'une plus forte précarité, c'est seulement parce que cette première expérience professionnelle ne correspond pas à un emploi de fonctionnaire $\left(6 \%\right.$ des $1^{\text {ers }}$ emplois obtenus en France par les diplômés de l'enseignement supérieur correspondent à des postes de fonctionnaires). En effet, comme les jeunes s'insérant en France, dans trois cas sur dix, les jeunes expatriés bénéficient d'un contrat de travail de type CDI (contrat à durée indéterminée). 
Enfin, dans un cas sur deux, la fin de l'emploi à l'étranger rime avec la démission du jeune. Ces jeunes abandonnent ainsi plus souvent leur premier emploi que les jeunes restés en France (46\% contre $38 \%$ ). Faut-il y voir la conséquence d'une décision de retour au pays?

\section{LES DÉTERMINANTS D'UNE PREMIÈRE EXPÉRIENCE PROFESSIONNELLE À L'ÉTRANGER}

L'étude des déterminants de la migration est réalisée en recourant à un modèle logistique. Cette modélisation est nécessaire à notre étude parce qu'elle permet d'examiner l'effet de chaque caractère indépendamment des autres (« toutes choses égales par ailleurs »). Cela permet d'identifier l'effet propre de chaque variable et de savoir, par exemple, que le fait d'avoir un père ou une mère cadre accrôt la propension à migrer du jeune, indépendamment de son niveau de diplôme, de son sexe, de sa situation familiale et de son expérience professionnelle (cf tableau 4). Compte tenu de la forte corrélation entre niveau de diplôme et âge au baccalauréat, une variable sur la réussite au baccalauréat n'a pas été introduite.

La mobilité géographique en début de vie active croît avec le niveau de diplôme et varie selon les spécialités de formation. «Toutes choses égales par ailleurs », les diplômés d'un troisième cycle scientifique et les jeunes issus d'une école de commerce ont la plus forte propension à réaliser une expérience professionnelle temporaire à l'étranger. Ils partent plus souvent que les titulaires d'un titre d'ingénieur et que les diplômés d'un troisième cycle de sciences humaines et sociales. Ce sont les diplômés d'un premier cycle ou second cycle scientifiques qui sont les moins enclins à l'expatriation. Si le phénomène migratoire s'amplifie très fortement avec le niveau de diplôme, n'est-ce pas parce que de telles caractéristiques permettent aux jeunes diplômés de mieux tirer profit de leurs années d'études ? En effet, les jeunes qui prospectent sur un marché du travail international, plutôt que national, souhaitent sans doute renforcer leurs chances de trouver un emploi conforme à leurs aspirations, en particulier en termes de revenus présents et futurs. Dans ce cas, le bénéfice attendu de la mobilité a de fortes chances de dépasser son coût financier et psychologique, ce coût pouvant lui-même décroître avec le niveau d'études dans la mesure où ils sont notamment plus aptes à voir une recherche d'emploi efficace. Par ailleurs, ne peut-on pas également voir dans ces résultats des effets d' " auto-sélection » positive ? Par ailleurs, que faut-il penser des différences entre spécialités ? La faible mobilité des diplômés des deuxièmes cycles scientifiques ou technologiques reflète sans doute leurs difficultés à accéder au marché de l'emploi scientifique et technologique réservé aux plus diplômés, et à valoriser leur formation dans d'autres secteurs.

La mobilité géographique d'insertion est également conditionnée par la mobilité géographique de formation (cf. tableau 4). Le fait d'avoir déjà migré peut développer, sinon un goût pour la migration, tout au moins une appréhension moins forte face à un départ à l'étranger dans la mesure où ils ont déjà eu l'opportunité de changer d'environnement. Ils sont sans doute également moins attachés à la région qui les a formés, et prêts à envisager des opportunités d'emplois plus dispersées dans l'espace. 
Tableau 4 : Les facteurs d'une expatriation pour une première expérience professionnelle

\begin{tabular}{|c|c|c|c|}
\hline & Coef. & Significativité & Odds ratio \\
\hline Constante & $-2,8738$ & $* * *$ & \\
\hline Homme & réf. & & \\
\hline Femme & 0,2151 & ns & 1,566 \\
\hline Situation familiale à la fin des études & & & \\
\hline Seul & réf. & & \\
\hline Avec un conjoint & 0,4399 & ns & 3,843 \\
\hline Sans enfant & réf. & & \\
\hline Avec des enfants & 0,0060 & ns & 1,984 \\
\hline $\begin{array}{l}\text { Âge à la fin des études ( } 1 \text { année } \\
\text { supplémentaire) }\end{array}$ & $-0,0215$ & ns & 1,035 \\
\hline Origine sociale & & & \\
\hline Parents non cadres & réf. & & \\
\hline Père ou mère cadre & 0,3086 & $* *$ & 1,716 \\
\hline Niveau et spécialité de formation & & & \\
\hline $1^{\mathrm{er}}$ cycle (DEUG, DEUST, BTS-DUT...) & $-1,7645$ & $* * *$ & 0,260 \\
\hline $2 \mathrm{~d}$ cycle maths, $\mathrm{sc}$, tech & $-1,8622$ & $* * *$ & 0,380 \\
\hline $\begin{array}{l}2 \mathrm{~d} \text { cycle LSH, gestion } \\
3^{\text {ème }} \text { cycle maths, sc, tech }\end{array}$ & $\begin{array}{l}-0,9677 \\
\text { réf. }\end{array}$ & $* * *$ & 0,563 \\
\hline $3^{\text {ème }}$ cycle LSH, gestion & $-0,9774$ & $* * *$ & 0,589 \\
\hline Écoles d'ingénieurs & $-0,5853$ & $* *$ & 0,892 \\
\hline Écoles de commerce & 0,0830 & ns & 1,477 \\
\hline $\begin{array}{l}\text { Mobilité géographique pendant les études } \\
\text { Pas de changement de région entre } 6^{\text {ème }} \\
\text { fin des études }\end{array}$ & réf. & & \\
\hline $\begin{array}{l}\text { Changement de région entre } 6^{\text {ème }} \text { et fin } \\
\text { des études }\end{array}$ & 0,3715 & $* * *$ & 1,830 \\
\hline Pas d'expérience prof. durant les études & réf. & & \\
\hline Expérience prof. durant les études & $-0,1354$ & ns & 1,146 \\
\hline$-2 \log L$ & 3260,8 & $* * *$ & \\
\hline $\begin{array}{c}\text { N } \\
\text { Somers D }\end{array}$ & 0,453 & $\begin{array}{l}21519 \\
\text { Concordant }\end{array}$ & $70 \%$ \\
\hline
\end{tabular}

Note : L'individu de référence est un jeune homme français célibataire sans enfant sortant d'un $3^{\text {ème }}$ cycle de sciences et sans expérience professionnelle. Son père ou sa mère ne sont pas cadres, il n'a pas changé de région entre la $6^{\text {ème }}$ et sa sortie du système de formation supérieure et il a obtenu son baccalauréat à l'heure.

Note de lecture : La probabilité de migrer d'un jeune qui sort d'un $1^{\text {er }}$ cycle est 4 fois moins importante que celle de l'individu de référence.

Source : « Génération 98 », Céreq 2001.

Un facteur agit sur les comportements migratoires : l'origine sociale. Toutes choses égales par ailleurs, avoir un père ou mère cadre s'avère favorable à une mobilité internationale de début de carrière. Cet effet pour les jeunes issus des milieux favorisés, peut notamment être induit par un soutien financier plus important au moment du déménagement ou par des réseaux de connaissances plus élargis territorialement ou lié à des perceptions familiales différentes de l'éloignement. En revanche, les compor- 
tements migratoires des hommes et femmes, des plus ou moins jeunes comme des célibataires et des jeunes en couple n'apparaissent pas significativement différents à ce stade de l'analyse.

\section{UNE EXPATRIATION PROFESSIONNELLE TEMPORAIRE EST-ELLE TOUJOURS SYNONYME DE CONDITIONS D'EMPLOI PLUS FAVORABLES QU'EN FRANCE?}

Parmi les diplômés de l'enseignement supérieur, les jeunes expatriés occupent plus fréquemment des emplois de cadres et bénéficient de salaires plus élevés qu'en France. Cependant, n'oublions pas que les jeunes ayant eu une expérience professionnelle à l'étranger sont nettement plus diplômés. Il n'est pas étonnant qu'ils accèdent plus facilement à des emplois plus qualifiés et bien rémunérés. Il est alors possible que les écarts observés en termes d'insertion soient liés à des caractéristiques différentes de nos deux sous populations - les migrants et les non migrants - C'est pourquoi, il est nécessaire de raisonner «toutes choses égales par ailleurs » et de recourir à une série de modélisations tenant compte de ces aspects (cf. annexe 1).

Les résultats de ces traitements statistiques permettent d'affirmer que considérée dans son ensemble, l'émigration favorise l'accès à des premiers salaires et à une première position professionnelle plus élevés (cf. tableau 5). Ainsi, à niveau de diplôme, sexe, âge et nature des entreprises, donnés, les jeunes ayant une première expérience à l'étranger bénéficient de meilleures rémunérations lors de leur embauche que leurs compatriotes s'insérant en France. Ces effets ne sont ainsi pas liés à des profils différents entre migrants et non migrants. Rappelons toutefois qu'il reste difficile d'associer ces salaires à des niveaux de vie plus relevés. En termes de statut professionnel, l'intérêt d'une première expérience professionnelle à l'étranger apparaît également réel, puisqu'ils occupent plus fréquemment des emplois de cadres lors de leur premier emploi (cf. tableau 5).

En revanche, une migration internationale temporaire se caractérise globalement par le fait que les jeunes expatriés ont moins souvent des emplois stables. Toutefois, ce résultat peut être soumis à controverses, dans la mesure où les contrats de travail à l'étranger sont décrits par les jeunes à partir d'une nomenclature s'appuyant sur la législation française du travail. Ne peut-on pas en effet considérer que la précarité est différente d'un pays à l'autre, sachant qu'elle est ici associée au fait que les jeunes n'ont pas de Contrats à Durée Indéterminée (CDI) ? N'existe-il pas un biais lié au fait que seuls les jeunes travaillant pour une entreprise française seraient susceptibles d'avoir de tels contrats de travail ? Malheureusement, nous ne disposons pas d'informations suffisantes dans l'enquête "Génération 98 » pour répondre à de telles questions.

Dans cette première confrontation des conditions d'emplois des migrants et des jeunes, il demeure difficile de prendre en compte les différentes destinations des diplômés de l'enseignement supérieur ayant une première expérience professionnelle à l'étranger, compte tenu de la taille de notre échantillon. Pourtant, ne peut-on pas 
Tableau 5 : Expatriation et qualité des premiers emplois

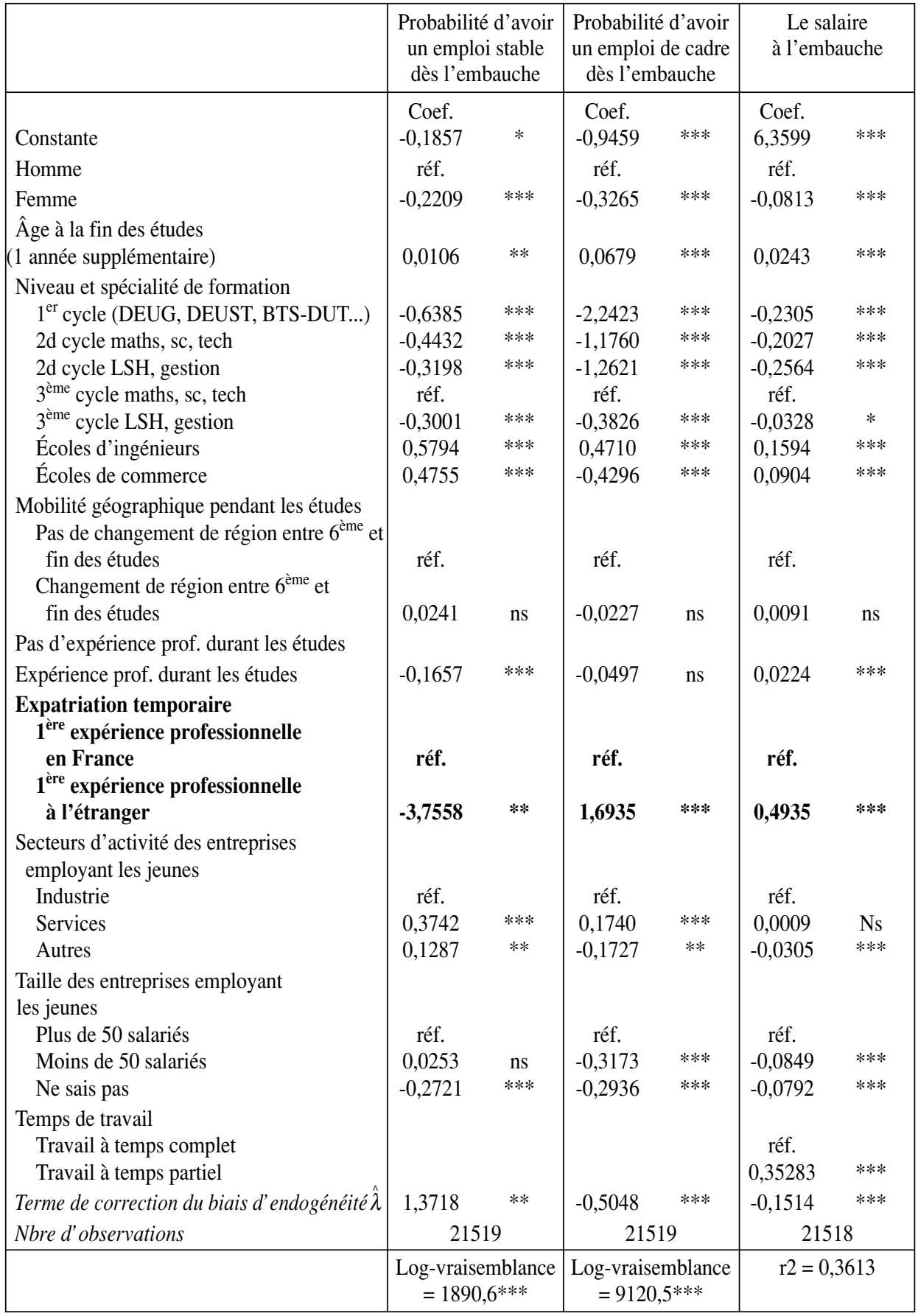

Note : *** significatif à $1 \%$, ** significatif à $5 \%$, * significatif à $1 \%$, ns non significatif. Source : « Génération 98 », Céreq 2001. 
considérer que les effets de la migration risquent d'être sensiblement différents d'un pays à un autre, ou d'une zone à l'autre ? En effet, l'examen des impacts des mobilités sur le territoire national pour les jeunes diplômés de l'enseignement supérieur lors de leur entrée dans la vie active (Perret et Roux, 2004) a permis de montrer que seules certaines mobilités géographiques professionnelles s'accompagnent d'une « prime à l'emploi »

\section{CONCLUSION}

Être présent sur le marché du travail national en ayant eu une première expérience professionnelle à l'étranger est un phénomène exceptionnel pour les jeunes diplômés français. Émigrer après ses études reste une démarche marginale pour les diplômés de l'enseignement supérieur français. Béret et al. (2003) ont, à cet égard, estimé que seuls $3 \%$ de ces jeunes ont eu au moins un emploi à l'étranger dans leurs premières années de vie active et que moins de $1 \%$ résident encore à l'étranger trois ans après la fin de leurs études. Les plus diplômés et les jeunes issus de filières scientifiques sont plus enclins à tenter leurs chances à l'étranger. Une migration professionnelle temporaire à l'étranger est intéressante pour les jeunes diplômés de l'enseignement supérieur puisqu'ils accèdent à des premiers emplois plus qualifiés et mieux payés. Faut-il y voir l'une des causes des départs des jeunes ? Ne peut-on pas supposer que ces résultats sont applicables à l'ensemble des jeunes — diplômés ou non - tentant leurs chances hors de nos frontières ? Par ailleurs, n'oublions pas que l'intérêt pour les diplômés de l'enseignement supérieur d'avoir une expérience professionnelle peut permettre des conditions d'emploi et de carrière en France plus favorables. En début de carrière, une expérience professionnelle à l'étranger est-elle synonyme de meilleure réussite professionnelle en France sachant qu'en outre, le retour au pays est considéré comme nécessaire (Gribble, 2008) ? La valorisation des déplacements transnationaux en termes de carrière professionnelle est sans doute un moteur essentiel à l'émergence d'un marché du travail transeuropéen et mérite donc de faire l'objet d'explorations. 


\section{Références bibliographiques}

AGBOSSOU Igor, CAREL Sophie et CARO Patrice (2007) Les échanges européens Erasmus... Accroître la mobilité des enseignants pour développer celle des étudiants, Bref, n ${ }^{\circ} 246$, pp. 14.

BRENNAN John (1993) Higher education quality: a European dimension in John Brennan and Frans Van Vught (eds.) Questions of Quality: In Europe and Beyond, QSC Higher Education Report, $n^{\circ} 1$.

CATTAN Nadine (2004) Genre et mobilité étudiante en Europe. Espace, Population et Sociétés, $\mathrm{n}^{\circ} 1$, pp. $15-27$.

CÉREQ (2002) Quand l'école est finie... Premiers pas dans la vie active de la Génération 98, CEREQ.

COMMISSION EUROPÉENNE (2000) Enquête sur la situation socio-économique des étudiants Erasmus, Direction générale de l'éducation et de la culture [en ligne]

http://europa.eu.int/comm/education/programmes/socrates/erasmus/survey_fr.pdf (page consultée le 12 septembre 2008)

FRANÇOIS-PONCET Jean (2000) Rapport d'information sur l'expatriation des jeunes Français, Rapport du Sénat, $\mathrm{n}^{\circ} 388$.

GENTIL Bernard (2003) La population française immatriculée à l'étranger est en forte hausse, INSEE Première, $\mathrm{n}^{\circ}$ 919, pp. 1-4.

GIRET Jean-François, MOLINARI-PERRIER Mickaëlle et MOULLET Stéphanie (2006) 20012004 : les sortants de l'enseignement supérieur face au marché du travail : enquête «Génération 2001 », Notes Emploi Formation, n² 21, 37 p.

GIRET Jean-François, MOULLET Stéphanie et THOMAS Gwenaëlle (2002) De l'enseignement supérieur à l'emploi : les trois premières années de vie active de la «Génération 98 », Notes Emploi Formation, $\mathrm{n}^{\circ} 1,43 \mathrm{p}$.

GRENNE William H. (1997) Econometric Analysis, Prentice Hall.

GRIBBLE Cate (2008) Policy options for managing international student migration: the sending country's perspective? Journal of Higher Education Policy and Management, vol. 30, $\mathrm{n}^{\circ} 1$, pp. 25-39.

HAUG Guy et TAUCH Christian (2001) Trends in Learning Structures in Higher Education (II), Follow up Report prepared for the Salamanca and Prague Conferences of March/ May 2001 Finnish National Board of Education.

RECOTILLET Isabelle (2007) PhD Graduates with Post-doctoral Qualification in the Private Sector: Does It Pay Off?, Labour, vol. 21, n 3, pp. 473-502.

JAHR Volker et TEICHLER Ulrich (2006) International Experience and Mobility of Graduates in Ulrich Teichler (ed.), Careers of University Graduates: Views and Experiences in Comparative Perspectives, Kluwer Academic Publishers.

MURPHY-LEJEUNE Elizabeth (2003) Un portrait d'étudiants mobiles en l'an 2000 : le cas français (2003) in Francine Vaniscotte, Aude Houguenague et Anne West (dir.) La Mobilité étudiante en Europe, mythe ou réalité ? Comparaison France-Royaume-Uni, L'Harmattan, pp. 39-90.

MURPHY-LEJEUNE Elizabeth (2005) L'étudiant européen voyageur, un nouvel étranger, Didier.

OVE-ORFS (2004) Les étudiants du PUN : séjours d'études et stages à l'étranger [en ligne], http://www.univ-

rouen.fr/servlet/com.univ.utils.LectureFichierJoint?CODE=1087459414865\&LANGUE=0 (page consultée le 12 septembre 2008)

PERRET Cathy, ROUX Valérie (2004) La mobilité géographique en début de carrière : un moteur de réussite ? », Formation Emploi, n 87, pp. 45-62. 
PICHON Laure-Anne, COMTE Maurice et POULARD Xavier (1999) Les étudiants en séjour d'études à l'étranger. Qui ? Pourquoi ? Comment ? : observation des étudiants ayant effectué un séjour d'études à l'étranger en 1997-98, Observatoire Universitaire Régional de l'Insertion Professionnelle.

SOLLOGOUB Michel et ULRICH Valérie (1999) Les jeunes en apprentissage ou en lycée professionnel, Économie et Statistique, ${ }^{\circ}{ }^{\circ} 323$, pp. 31-52.

TEICHLER Ulrich et JANSON Kerstin (2007) The Professional Value of Temporary Study in Another European Country: Employment and Work of Former ERASMUS Students, Journal of Studies in International Education, vol. 11, n 3-4, pp. 486-495.

TREMBLAY Karine (2005) Academic Mobility and Immigration, Journal of Studies in International Education, vol. 9, $\mathrm{n}^{\circ}$ 3, pp. 196-228.

PAPATSIBA Vassiliki (2003) Des étudiants européens. Erasmus et l'aventure de l'altérité, Peter Lang.

WOOLRIDGE Jeffrey M. (2002) Econometric Analysis of cross section and panel data, MIT Press. 


\section{ANNEXE 1 : Les rendements comparés du premier emploi : méthode de mesure}

Trois indicateurs sont utilisés pour étudier le rendement comparé au premier emploi pour les expatriés et les jeunes travaillant en France :

- le salaire net à l'embauche (primes incluses) exprimé en logarithme ;

- la probabilité d'avoir un emploi de cadre dès l'embauche notée Cadre $\left(\mathrm{Cadre}_{\mathrm{i}}=1\right.$ si l'individu i occupe un emploi de cadre, 0 sinon);

- la probabilité d'avoir un emploi stable dès l'embauche ; la stabilité étant appréciée par le fait d'avoir un statut de fonctionnaire ou un Contrat à Durée Indéterminée (CDI), notée $\mathrm{CDI}\left(\mathrm{CDI}_{\mathrm{i}}=1\right.$ si l'individu i bénéficie d'un contrat CDI ou fonctionnaire, 0 sinon $)$.

La mesure de l'effet de la migration sur l'insertion professionnelle des jeunes, c'est-àdire la comparaison des conditions de premier emploi des expatriés et des sédentaires est un phénomène complexe. En effet, conditions d'emploi et migration peuvent être inter-connectées et il est difficile de faire la part entre les causes et les conséquences : le jeune migre-t-il parce qu'il a trouvé un emploi de meilleure qualité ou trouve-t-il un emploi de meilleure qualité parce qu'il a migré ? Migration et conditions de premier emploi peuvent alors être des variables endogènes et ne pas être indépendantes : la migration étant souvent liée à l'obtention d'un emploi de tel ou tel type et/ou l'obtention d'un emploi de tel ou tel type dépendant de la migration. La migration n'apparaît également pas comme un phénomène aléatoire car il se peut qu'il existe une sélection dans les individus migrants fondée en partie sur des caractéristiques non observées qui peuvent également conditionner les conditions de premier emploi. Par exemple, si les individus qui migrent ont les caractéristiques inobservables les plus favorables en termes d'insertion professionnelle (motivation, relationnelle aisée, etc.), il y a alors un risque d'attribuer à tort l'effet de ces variables non observées à la migration. On se retrouve donc confronté à un biais de sélection qui rend la migration endogène à l'insertion.

Pour estimer l'effet spécifique de l'expatriation temporaire, la stratégie adoptée pour contrôler ce problème d'endogénéité est de recourir à une méthode économétrique plus complète que celle consistant à introduire directement la variable migration (MIG avec $\mathrm{MIG}_{\mathrm{i}}=1$ si le jeune s'est expatrié, 0 sinon) au sein d'une régression logistique ou d'une régression linéaire dans le cas du salaire. Il s'agit ainsi de procéder à une estimation en deux étapes, l'une dans laquelle on s'intéresse au processus qui a généré la migration, permettant en seconde étape de purger la variable migration de son endogénéité présumée. À cet effet, on applique la méthode de Barnow, Cain et Goldberger (1981) qui permet de tester l'hypothèse d'endogénéité du choix de la migration dans les équations relatives à la qualité de l'emploi (position professionnelle et stabilité) et au salaire, et de récupérer le rendement réel de la migration une fois cette endogénéité prise en compte.

La première étape consiste ainsi à modéliser à partir d'un modèle probit la probabilité de migrer en fonction d'un ensemble de variables explicatives exogènes. Cette probabilité de migrer est déterminée par l'équation suivante :

(1) $M I G^{*}=X_{1} \alpha+u$

avec

$$
\begin{cases}M I G=1 & \text { si } M I G^{*}>0 \\ M I G=0 & \text { si } M I G^{*} \leq 0\end{cases}
$$


La seconde étape consiste à modéliser la probabilité d'être en emploi stable et la probabilité d'avoir un emploi de cadre à partir d'une équation probit, ainsi que le salaire avec une équation linéaire (OLS) du type :

(2) $Q^{*}=X_{2} \beta_{1}+M I G \alpha_{1}+\hat{\lambda} \delta_{1}+\vartheta$

Avec $\mathrm{Q}^{*}=\mathrm{CDI}^{*}$ ou Cadre* ou $\log$ (salaire).

$\hat{\lambda}$ est le terme correcteur du biais d'endogénéité : il prend en compte la corrélation entre les résidus de l'équation [1] et ceux de l'équation [2]. Il permet d'obtenir une estimation non biaisée du coefficient $\forall_{1}$. On calcule $\hat{\lambda}$ selon la méthode de Barnow, Cain et Goldberger (1981), comme suit :

$$
\begin{aligned}
& \lambda=E\left[\vartheta / X_{2}, M I G\right]=M I G \times E\left[\vartheta / X_{2}, M I G=1\right]+(1-M I G) \times E\left[\vartheta / X_{2}, M I G=0\right] \\
& \hat{\lambda}=M I G \times \frac{\phi\left(\hat{\alpha} X_{1}\right)}{\Phi\left(\hat{\alpha} X_{1}\right)}+(1-M I G) \times \frac{-\phi\left(\hat{\alpha} X_{1}\right)}{1-\Phi\left(\hat{\alpha} X_{1}\right)}
\end{aligned}
$$

$\phi$ et $\Phi$ sont respectivement la fonction de densité et la fonction distributive de la loi normale standard. Le coefficient $\forall_{1}$ donne le réel impact de la migration ; le coefficient $\delta_{1}$ montre dans quelle mesure certaines caractéristiques inobservables qui influencent la migration conditionnent également la qualité du premier emploi. 